\section{$\mathbf{m} / \mathbf{s}$}

médecine/ sciences $2001 ; 17: 687-90$

\title{
À NOUVEAU MILLÉNAIRE NOUVELLE MICROBIOLOGIE ?
}

\section{Philippe Sansonetti}

\section{ADRESSE}

$P$. Sansonetti : U nité de pathogénie microbienne moléculaire et Inserm U. 389, Institut Pasteur, 28, rue du Docteur-Roux, 75724 Paris Cedex 15, France.

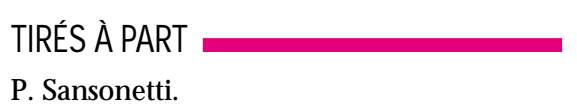

$\mathbf{F}$ aut-il encore en 2001 proposer un titre accrocheur pour attirer l'attention de la communauté scientifique sur la microbiologie? Cette discipline fait-elle toujours figure de parent pauvre face aux disciplines reines comme la biologie cellulaire, la biologie du développement et la neurobiologie ? Étant toujours dans le doute, j'y ai cédé... probablement à tort...

\section{II n'y a pas de nouvelle microbiologie mais une microbiologie qui se renouvelle}

II y a, semble-t-il, deux manières de faire reconnaître la microbiologie, I'une défensive, ressassant à qui veut encore l'entendre d'évidentes ritournelles telles: «la biologie moléculaire et l'immunologie sont nées de la microbiologie », " on connaît à peine $1 \%$ des espèces bactériennes existant sur la planète », «les maladies infectieuses causent le tiers des décès observés dans le monde, d'anciennes ré-émergent et de nouvelles émergent », etc. Ces arguments ont un impact sur les médias, le public et parfois les politiques, peu sur les scientifiques eux-mêmes, particulièrement sur les jeunes face à leurs choix de carrière. II existe une autre manière, appelons-la offensive, consistant pour les microbiologistes eux-mêmes à faire la preuve qu'ils renouvellent leur discipline et savent la rendre attractive. Cette "nouvelle microbiologie », évidente pour qui suit la littérature spécialisée, a clairement montré son visage à l'occasion de la réponse aux récents appels d'offres du Programme de recherche fondamentale en microbiologie, maladies infectieuses et parasitaires du ministère de la Recherche*. C'est la démonstration d'une microbiologie créative, dynamique, pragmatique, concrète, ouverte aux autres disciplines fondamentales, s'en nour- rissant et leur fournissant réciproquement outils et modèles pertinents d'étude. C'est de sa capacité extraordinaire d'absorption des autres disciplines fondamentales: génomique, biologie structurale, biologie cellulaire, immunologie, que la microbiologie tire son actuelle vitalité. C'est en résumé dans l'ouverture et la transdisciplinarité que la microbiologie, au lieu de perdre son identité, a trouvé les germes de sa renaissance. Exemple à mûrir...

\section{Le paradigme de la pathogénicité des infections bactériennes}

S'il est un domaine caractéristique de cette «nouvelle microbiologie», c'est celui de l'étude de la pathogénicité microbienne, particulièrement bactérienne qui a, en l'espace de 25 ans, subi plusieurs évolutions (révolutions ?) [1]. Partant d'une approche essentiellement descriptive, reposant sur l'analyse de modèles animaux d'infection, elle a intégré, dès la fin des années 1970, la biologie moléculaire. Ce furent le clonage des gènes de capsules, de toxines, de facteurs d'adhérence, d'invasion et de survie intracellulaire, la reconnaissance de leur localisation sur des plasmides, des bactériophages, voire même en bloc sur le chromosome, préfigurant le concept à venir d'îlots de pathogénicité (PAI), la production de mutants et leur complémentation, la réactualisation des postulats de Koch, pour l'occasion rebaptisés postulats moléculaires de Koch [2]. La physiopathologie moléculaire des infections était apparue, mais la diversité de mutants et transformants engendrés dans un nombre croissant d'espèces pathogènes n'était plus à l'échelle d'une analyse in vivo. Ainsi,

* Philippe Sanson etti est président du comité scientifiquedu programme derecherchefondamentaleen microbiologie, maladies infectieu ses et parasitaires. 
dès le milieu des années 1980, une course s'engagea pour mettre au point une batterie de modèles cellulaires, cette approche in vitro s'avérant plus compatible avec le criblage de banques de plusieurs milliers de clones, quitte à revenir secondairement à des études in vivo. Durant cette même période, il est clairement apparu que l'expression des gènes de virulence était très étroitement réglée dans le temps et l'espace, particulièrement sous l'influence de conditions correspondant à l'environnement du micro-organisme au sein de l'hôte (température, $\mathrm{pH}$, osmolarité, concentration de certains ions). On développa même des techniques permettant d'identifier des gènes sélectivement exprimés in vivo [3]. Un peu plus tard devait apparaître la notion de quorum sensing dans le cadre duquel la bactérie règle l'expression de certains de ses gènes, y compris de pathogénicité, en fonction de la perception de sa propre densité au sein d'une niche donnée [4]. La pathogénicité n'était donc pas seulement affaire de gènes de virulence, elle était aussi hiérarchisation et orchestration de leur expression, évitant une cacophonie pénalisante pour une manifestation optimale du pouvoir pathogène. Mieux vaut ne pas commencer par tuer la cellule dans laquelle on doit élire domicile...

L'observation quotidienne de l'interaction microbes-cellules afin d'analyser le phénotype des mutants étudiés mena vers la fin des années 1980 à prêter une attention croissante à la biologie de la cellule cible elle-même [5]. L'introduction de l'observation par microscopie confocale de «l'interface microbe-cellule », après marquage du microbe et de composants cellulaires par des sondes fluorescentes, fut soudain comme l'ouverture d'un atlas de biologie cellulaire. Avec l'aide de collègues toxinologistes qui «flirtaient » depuis des années avec la biologie cellulaire, puis de biologistes cellulaires motivés parfois par la recherche chez les procaryotes d'outils innovants pour l'analyse des eucaryotes, la description devint analyse et ce qui avait commencé comme la mise au point de modèles simples criblage de mutants/ transformants, devint analyse approfondie, morphologique, génétique et biochimique, des interactions entre effecteurs microbiens et récepteurs cellulaires. Les pathogènes invasifs qui ont développé un programme exhaustif d'interactions avec les cellules eucaryotes jouèrent de fait un rôle moteur dans l'évolution de cette approche. La symbiose microbiologie-biologie cellulaire s'était faite, la microbiologie cellulaire était née [6].

Dès le début des années 1990, la situation avait donc considérablement évolué, on avait acquis un sens de ce que représentait la pathogénicité en termes génétiques et moléculaires, on avait réalisé le rôle essentiel du transfert horizontal de blocs de gènes dans la " construction d'un pathogène » [7], notion qui allait être spectaculairement confirmée et amplifiée par la séquence complète et l'annotation du génome de plusieurs de ces pathogènes, on avait compris qu'un pathogène ne se manifestait comme tel que parce qu'il était capable de percevoir son environnement et de s'y adapter, on avait enfin amené l'analyse de l'infection à un niveau de définition et de résolution jamais atteint, grâce à l'introduction de la biologie cellulaire. Durant les années 1990, ces différents secteurs $n$ 'ont fait que s'amplifier avec une analyse toujours plus en profondeur des mécanismes moléculaires de signalisation et une large extension du spectre des micro-organismes analysés: pathogènes de mammifères, d'insectes, de plantes. L'outil moléculaire et la communauté de certains mécanismes de pathogénicité avaient aussi contribué à rapprocher des secteurs et des acteurs de la microbiologie qui, antérieurement, ne se fréquentaient guère.

\section{Et maintenant ?}

L'avenir ne se prédit heureusement pas en sciences, pas même en microbiologie... II est en revanche intéressant de tenter d'identifier les mouvements qui sous-tendront les progrès à venir. Loin de l'exhaustivité, six grands défis peuvent être proposés: intégrer la génomique, ou comment gérer la quantité? Intégrer plus encore les approches fondamentales, ou comment gérer la transition vers une microbiologie subcellulaire ? Sortir de l'in vitro réductionniste et aller vers l'in vivo, ou comment gérer la transition vers une microbiologie tissulaire ? Intégrer plus avant l'étude de la réponse de l'hôte et développer l'étude des bases génétiques et moléculaires de sa sensibilité/ résistance aux infections, ou comment aborder la diversité ? Élargir les recherches à l'étude du mode de vie environnemental des pathogènes, ou comment analyser la globalité ? Étudier «en miroir » les micro-organismes ayant un effet bénéfique sur l'hôte, ou comment analyser symbiose et commensalisme?

\section{Pathogénicité et post-génome}

Nous avions jusqu'à présent procédé à une chasse à l'information génétique, nous pressentons que de chasseurs, nous sommes devenus gibiers d'un fantastique système à produire de l'information qui nous poursuit et nous obsède ; en effet, la séquence et I'annotation des génomes d'un nombre croissant de pathogènes, mais aussi de leurs hôtes naturels, vecteurs ou modèles (homme, souris, moustique, plante/ Arabidopsis), les techniques de la génomique, en particulier l'étude des transcriptomes et protéomes des pathogènes et de leurs hôtes «au repos» et en situation d'infection, I'utilisation de techniques tel le double hybride à l'écheIon génomique appliquées à la recherche de cibles eucaryotes d'effecteurs microbiens, engendrent une masse d'information jamais jusqu'alors disponible et même si les approches in silico se développent de façon spectaculaire, cette bio-informatique n'éliminera pas la nécessité de valider les données obtenues dans des modèles biologiques pertinents in vitro et in vivo. Serons-nous en mesure d'organiser et d'utiliser autant d'informations et de les insérer dans de grands schémas de pathogénicité ? La réponse ne peut être que positive, l'originalité et la créativité scientifiques, par touches successives, composeront ce nouveau paysage, le temps et les avancées technologiques feront le reste. 


\section{Transition vers la microbiologie subcellulaire}

Une partie du succès résidera dans notre capacité d'identifier efficacement les cibles eucaryotes des effecteurs microbiens. La connaissance croissante des composants structuraux et de signalisation eucaryotes engagés dans la motilité cellulaire, dans le trafic et la maturation des compartiments intracellulaires, dans les voies de signalisation contrôlant le cycle ou la mort cellulaire, dans la transcription et les voies de signalisation impliquées dans le développement augmentent nos chances d'identifier les cibles pertinentes des effecteurs microbiens. Le développement de systèmes rapides d'analyse in vitro permettant d'identifier activation ou inactivation de ces cibles potentielles sera essentiel. Une autre clé du succès résidera dans notre capacité de décrypter le détail des interactions moléculaires engagées entre les effecteurs bactériens et leurs cibles eucaryotes. Pour ce faire, la biologie structurale, déjà largement utilisée pour l'analyse des rapports structure-fonction des toxines, devra s'intégrer de façon routinière dans nos approches.

\section{Transition vers la microbiologie tissulaire}

II y a aussi loin de la microbiologie cellulaire à la microbiologie tissulaire que de l'infection à la maladie infectieuse. Comment combler l'espace qui les sépare ? Beaucoup a d'ores et déjà été fait du côté des procaryotes, particulièrement par la mise au point de méthodes de criblage négatif de mutants comme la méthode de signature tagged mutagenesis (STM [8]). Beaucoup reste à faire du côté de I'hôte. Le problème n'est pas simplement technique, il est aussi culturel, conceptuel et éthique. II ne peut y avoir d'appréhension de la globalité d'un processus infectieux sans une approche physiologique et transdisciplinaire, sans un raisonnement de médecine expérimentale. C'est à une synthèse de I'héritage de Pasteur, Koch, Metchnikov, Erlich et Claude Bernard qu'il convient de nous livrer, deux handicaps majeurs entravant actuellement son obtention : I'insuffisance de l'enseignement et de la pratique de la physiologie et, liée ou non, la démédicalisation de la recherche dans les sciences du vivant en général et les maladies infectieuses en particulier. II semble important que s'amplifient deux mouvements synergiques: I'un allant de l'in vitro vers l'in vivo grâce à la mise en place de modèles cellulaires complexes mimant des structures intégrées cibles de pathogènes telles les grandes «barrières » de l'organisme : barrière épithéliale intestinale, barrière hémato-encéphalique, barrière alvéolaire pulmonaire, barrière cutanée, etc. Dans un esprit similaire, il est actuellement difficile de prédire la place exacte que prendront les modèles «animaux » alternatifs tels Caenorhabditis elegans [9] et la drosophile [10], leur principal intérêt étant la «facilité » de leur manipulation génétique. L'autre mouvement va de I'in vivo vers l'in vitro, permettant à partir de l'analyse des processus infectieux par des méthodologies innovantes combinant chirurgie expérimentale, microscopie intravitale et bi(multi)-photonique, histopathologie moléculaire et imagerie de pointe, d'apporter un niveau de définition et de résolution à l'échelle de la cellule infectée au sein du tissu, facilitant en retour la modélisation in vitro des processus. Cette analyse doit largement intégrer la réponse immunitaire, en particulier innée, composante majeure de l'expression des symptômes cliniques et paracliniques de l'infection. Plus que jamais, microbiologistes et immunologistes doivent étroitement collaborer. II est probable que la souris deviendra le modèle quasi exclusif de ces études, certes pour des raisons éthiques et financières, mais aussi et surtout du fait de la disponibilité de réactifs, d'animaux immunodéprimés permettant repopulation cellulaire et xénotransplantation de tissus hétérospécifiques, de souris génétiquement invalidées ou transgéniques, de la connaissance du génome permettant l'analyse plus ou moins globale du transcriptome de I'animal au niveau d'un tissu donné, en présence d'un agent infectieux. II convient d'adapter au modèle-souris les méthodolo- gies les plus innovantes en matière d'imagerie afin de développer, dans le temps et l'espace, une analyse non invasive du développement des processus infectieux. Celles-ci permettront non seulement d'éviter une approche in vivo jusqu'à présent essentiellement vivisectionniste, mais aussi de pallier l'aspect stochastique du développement des processus infectieux, particulièrement aux phases précoces. La détection transcutanée, par des caméras ultrasensibles, de la fluorescence de micro-organismes produisant un fluorochrome [11] ou d'organes/ tissus au sein desquels l'activation d'un promoteur d'intérêt entraîne l'expression d'une protéine hybride comportant un fluorochrome ou une enzyme comme la luciférase, doit permettre d'établir une cartographie très précise, en temps réel, du développement de l'infection [12]. De même, l'imagerie par résonance magnétique (IRM) semble appelée à jouer un rôle majeur dans le suivi des infections et de la réponse de l'hôte. L'introduction de substances paramagnétiques libérées spécifiquement au site infectieux devrait permettre d'introduire le paramètre "spécificité » indispensable à ces approches [13] .

\section{Intégrer l'étude de la réponse de l'hôte et développer l'étude des bases génétiques et moléculaires de sa sensibilité/ résistance aux infections}

Inutile de ré-insister sur l'importance et l'indissociabilité du couple microbiologiste-immunologiste dans l'analyse des processus infectieux. Au-delà cependant de l'analyse de la réponse innée et spécifique à un pathogène et de l'intrication de ces deux réponses, il est d'ores et déjà nécessaire de réfléchir, en coordination avec épidémiologistes et généticiens, à l'établissement de modèles in vitro et in vivo permettant d'identifier les gènes de l'hôte modulant sa susceptibilité d'espèce et d'organe aux infections, l'intensité et la sévérité de sa réponse qui conditionne la gravité, voire la létalité de l'infection. Le chemin a été montré pour les parasites intracellulaires comme les mycobactéries. La génomique des micro-orga- 
nismes et de leurs hôtes va faire rapidement exploser cette discipline avec, on l'espère, son cortège d'applications thérapeutiques et préventives.

\section{Élargir les recherches à l'étude du mode de vie environnemental des pathogènes}

De récentes épidémies de légionellose nous rappellent que les micro-organismes pathogènes ont une vie hors de leur hôte humain ou animal; pour Legionella pneumophila, par exemple, un macrophage alvéolaire pulmonaire n'est probablement pas perçu différemment d'un protozoaire hôte intermédiaire (Acanthamoeba, $\mathrm{H}$ artmanella) dans un système de distribution d'eau représentant la niche réelle de cette espèce. II est possible que dans de nombreux cas, les conditions de vie environnementales aient représenté la pression sélective responsable de la constitution du capital de gènes de pathogénicité. La génomique d'Escherichia coli nous a appris que les isolats pathogènes de cette espèce ( $p a t h o-$ vars) présentaient un génome de taille $20 \%$ ( 1 mégabase) supérieure à celle des formes commensales. II est maintenant essentiel de définir dans ce pool génétique additionnel ce qui revient à la pathogénicité, à la survie environnementale et quel est le recouvrement réel entre ces deux pools de gènes. II convient donc d'être dès maintenant aussi créatifs dans la modélisation in vitro de niches écologiques environnementales que nous l'avons été pour la pathogénicité. C'est peut-être aussi d'une vision globale du cycle de vie d'un pathogène que naîtront des stratégies de prévention innovantes. C'est particulièrement vrai pour les pathogènes entériques dans les zones sousdéveloppées, pour les pathogènes responsables de toxi-infections alimentaires et pour les pathogènes nosocomiaux dans l'environnement complexe de nos hôpitaux modernes [14].

\section{Étudier les micro-organismes ayant un effet bénéfique sur l'hôte, ou comment analyser symbiose et commensalisme}

Plus nous apprenons sur les patho690 gènes qui n'interviennent en fait que de façon accidentelle, plus nous mesurons la faiblesse de nos connaissances sur les micro-organismes symbiotiques et commensaux constitutifs de nos flores cutanées et muqueuses qui donnent pourtant lieu à des interactions permanentes et hautement organisées et réglées. Au-delà de la classique notion "d'effet de barrière » de ces flores protégeant les surfaces muqueuses contre la colonisation par des pathogènes, il apparaît de plus en plus clairement que les flores participent au développement, à la différenciation et à l'homéostasie des muqueuses elles-mêmes [15]. Peut-être dans ce secteur va se créer une nouvelle interface entre microbiologie et biologie du développement. Une perturbation de cette symbiose pourrait donner lieu à des phénomènes pathologiques: inflammation bucco-dentaire, intestinale, etc. II est donc temps que la créativité qui a été dévolue à l'étude des flores pathogènes s'applique à l'analyse des «bonnes flores ».

\section{Conclusions}

Si la réflexion offerte ci-dessus est par nature biaisée vers la bactériologie, il est clair que la plupart des domaines et tendances évoqués sont valables pour l'évolution des autres disciplines de la microbiologie: mycologie, virologie et parasitologie. Pour ce qui concerne les parasites, par exemple, les possibilités récentes d'analyse génétique de pathogènes majeurs tels que Plasmodium, Toxoplasma, et Leshmania, l'introduction de la biologie cellulaire dansl'analyse physiopathologique et la disponibilité attendue de plusieurs génomes vont révolutionner cette discipline. La microbiologie entre donc dans le millénaire, riche de promesses d'avancées cognitives exceptionnelles, mais aussi d'applications probables dans les domaines du diagnostic, de la thérapeutique et des vaccins. Nous avons voulu illustrer, par quatre articles écrits par d'éminents spécialistes de notre discipline, un certain nombre des approches et concepts qui font cette «nouvelle microbiologie». Microbiologie cellulaire, toxinologie moléculaire, analyse fonctionnelle exhaustive des génomes, parasitologie moléculaire et cellulaire, nous ont semblé être des thématiques susceptibles de convaincre la communauté scientifique que les microbiologistes jouaient bien dans la cour des grands

\section{RÉFÉRENCES}

1. Sansonetti PJ. Microbial pathogenesis: new paths into a new millenium. Trends $M$ icrobiol $2000 ; 8$ : 196-7.

2. Finlay BB, Falkow $S$. Common themes in microbial pathogenicity revisited. M icrobiol Mol Biol Rev 1997; 61 : 136-69.

3. Mahan MJ, Slauche JM, Mekalanos JJ. Selection of bacterial virulence genes that are specifically induced in host tissues. Science 1993 ; 259: 686-8.

4. Bassler BL. How bacteria talk to each other : regulation of gene expression by quorum sensing. Curr Opin M icrobiol 1999; 2 : 582-7.

5. Sansonetti PJ. Genetic and molecular bases of epithelial cell invasion by Shigella. Rev Infect Dis 1991 ; 13 : S285-92.

6. Cossart P, Boquet P, Normark S, Rappuoli R. Cellular Microbiology emerging. Science 1996 ; 271 : 315-6.

7. Hacker J, Kaper JB. The concept of pathogenicity islands. In : Kaper JB, H acker J, eds. Pathogen icity islands and other mobile virulence elements. Washington DC : ASM Press, 1999: 1-11.

8. Hensel M, Shea JE, Gleeson C, Holden DW. Simultaneousidentification of bacterial virulence genes by negative selection. Science $1995 ; 269: 400-3$.

9. Chauvet $S$, Ewbank JJ. Caenorhabditis ele gans: un modèle d'étude des interactions hôte-organisme pathogène. M ed Sci 2000 ; $16: 912-6$.

10. Imler JL, H offmann JA. Signaling mechanisms in the antimicrobial host defense of Drosophila. Curr O pin M icrobiol $2000 ; 3$ : 16-22.

11. Contag CH, Contag PR, Mullins JI, Spilman SD, Steven son DK, Benaron DA. Photonic detection of bacterial pathogens in living hosts. M ol M icrobiol 1995 ; 18 : 593-603.

12. Yang M, Baranov E, M oossa AR, Penman $\mathrm{S}, \mathrm{H}$ offman $\mathrm{RM}$. Visualizing gene expression by whole-body fluorescence imaging. Proc Natl A cad Sci USA 2000; 97 : 12278-82.

13. Louie AY, Meade TJ. Recent advances in $M R I$ : novel contrast agents shed light on in vivo biochemistry. New technologies for life sciences: a Trends Guide. T rends Biochem Sci 2000 ; S1471-931: 7-11.

14. Brun-Buisson C, Girou E. Les infections nosocomiales: bilan et perspectives. M ed Sci $2000 ; 16: 892-9$.

15. H ooper, LV, Falk PG, Gordon JI. Analyzing the molecular foundations of commensalism in the mouse intestine. Curr Opin M icrobiol $2000 ; 3$ : 79-95. 